\title{
David Goldenberg and Bradley J. Goldstein: Handbook of Otolaryngology, Head and Neck Surgery
}

\author{
Thieme, Stuttgart, 2010, 774 pp, ISBN 978-1-60406-928-7, \$100 USD
}

\section{Leo Clodius}

Received: 15 January 2011 / Accepted: 15 January 2011 /Published online: 16 February 2011

(C) Springer-Verlag 2011

Another book? Why? Its purpose: this pocket-sized volume is produced to provide the clinician with information helpful in the care of their patients. It decreases the amount of time searching for needed information, thus accelerating clinical care. The provided information is clinically most useful.

The authors, directors of otolaryngology and head and neck services in the USA, have published this handbook with what might be too many a new publishing house in Europe: Thieme in Stuttgart. Who is Thieme? They publish 137 peer-reviewed journals, including the field of plastic and reconstructive surgery, and annually, over 500 new scientific books.

This flexible handbook, designed to fit into the readers lab coat pocket, can be carried at all times, is both a comprehensive source of valuable information and a convenient portable clinical reference. The expert authors provide detailed and concise information on rhinology, otology, audiology, the thyroid and parathyroids, head and neck tumors, and facial plastic and reconstructive surgery. Seven sections divide the contents - general otolaryngology and perioperative care (including diagnostic imaging of the head and neck, anesthesia), TM joint disorders, robotic-assisted head and neck surgery, otology, rhinology, laryngology and the upper aerodigestive tract, the head and neck including anatomy, emergencies, chemotherapy, radiotherapy, and various different skin cancers. In addition, there are 64 pages on facial plastic and reconstructive surgery contributed by 26 authors. There are three additional appendices: basic procedures and methods of investigation, the cranial nerves, and otolaryngologic emergencies. A very helpful, detailed index of 77 pages concludes the text.

Each chapter, addressing a different clinical problem, begins with a list of key features and a section on epidemiology. Next, signs and symptoms, differential diagnosis, explanations of how to conduct the physical examinations, laboratory investigations, treatment options, outcomes, and follow-up.

This user-friendly handbook enables rapid reference also in difficult situations. The numerous cross-references and the extensive index allows for quick and easy finding of the needed information.

Every resident, fellow, and clinician, with patients in the vast field of head, neck, and facial surgery will certainly enjoy this clinical guide - a wonderful contribution.

L. Clodius $(\square)$

Zürich, Switzerland

e-mail: eurjplastsurg@juno.com 